\title{
Heat Capacity of Gaseous Perfluoropropane
}

\author{
Joseph F. Masi, Howard W. Flieger, Jr., and John S. Wicklund
}

\begin{abstract}
An accurate flow calorimeter, previously described, has been used to measure the heat capacity of gaseous perfluoropropane $\left(\mathrm{C}_{3} \mathrm{~F}_{8}\right)$ at three pressures up to 1.5 atmospheres at each of the temperatures $-30^{\circ},+10^{\circ},+50^{\circ}$, and $+90^{\circ} \mathrm{C}$. The results, believed accurate to 0.1 percent, have been extrapolated to zero pressure at each temperature, in order to obtain the ideal-gas heat capacity $\left(C_{p}^{0}\right)$ and the pressure coefficient of heat capacity. Values of $C_{p}^{0}$ are $31.10,34.22,37.02$, and 39.49 calories mole ${ }^{-1}$ degree $\mathrm{C}^{-1}$, respectively, at the four temperatures of measurement.
\end{abstract}

\section{Introduction}

Experimental gas heat capacities, obtained at several low pressures and over a range of temperatures, can be helpful in interpreting molecular structure and spectroscopic data and in studying equations of state at low pressure. The present investigation was undertaken as part of a comprehensive study of the thermodynamic and structural properties of fluorocarbons. The heat capacities of gaseous hexafluoroethane ${ }^{1}$ and perfluorocyclobutane ${ }^{2}$ have previously been reported.

\section{Experimental Procedure}

\subsection{Material}

A specially purified sample of perfluoropropane $\left(\mathrm{C}_{3} \mathrm{~F}_{8}\right)$ was very kindly furnished by the Minnesota Mining \& Manufacturing Corp. The entire sample was evaporated in a closed system while surrounded with a mixture of carbon tetrachloride and chloroform, cooled with dry ice to about $2 \mathrm{deg}$ below the normal boiling point of $\mathrm{C}_{3} \mathrm{~F}_{8}\left(-38^{\circ} \mathrm{C}\right)$. The vapor was condensed in a trap at liquid-nitrogen temperature. During this distillation the system was continuously pumped with a high-vacuum apparatus. First and last portions (about $25 \mathrm{~g}$ each) of the distillate were discarded. There was obtained a sample of about $450 \mathrm{~g}$ for use in the experiments.

A mass-spectrograph analysis of the sample, performed after most of the heat-capacity data had been taken, showed only a trace (less than 0.1 percent) of $\mathrm{CF}_{2} \mathrm{Cl}^{+}$and $\mathrm{CF}_{2} \mathrm{H}^{+}$ions. A gravimetric determination of the amount of carbon dioxide and moisture present was made by passing the entire flow-calorimeter sample, as vapor, through tubes of Anhydrone and Ascarite. Absorption of less than 0.01 percent was obtained. It was indicated that impurities should affect the heat-capacity measurements by much less than 0.1 percent.

\subsection{Apparatus and Method}

The construction and operation of the flow calorimeter have been described in detail ${ }^{3}$ a résumé has also been published. ${ }^{4}$

1 J. S. Wicklund, H. W. Flieger, Jr., and J. F. Masi, J. Research NBS 51, 91 (1953) R P2437.

, Chem Soc, 75,5082 (1953)

I. Petkof, J. Research N BS 48, 179 (1952) R P2303.

4 J F. Masi, J. Am. Chem. Soc. 74, 4738 (1952).
The heat capacity was determined at a number of different flow rates at each temperature and pressure, so that the effect of any residual heat leak could be eliminated by extrapolation to the zero of reciprocal rate. "Blank" determinations were made at several rates at each temperature and pressure to measure the amount of cooling, $\delta T$, experienced by the gas in passing through the calorimeter when no heat was applied. A total of 57 heat-capacity experiments and 67 blank experiments was made.

\section{Results}

The important data for all of the heat-capacity experiments are given in table 1 . The values of heat capacity, $C$, (column 7) were calculated from the power, $W$; the flow rate, $F$; the rise in temperature, $\Delta T$; and the temperature fall, $\delta T$, in the corresponding blank experiment, by the relation

$$
C=\frac{W}{F(\Delta T+\delta T)} \cdot
$$

These have been corrected by the amounts in column 8 in order to bring all results to the nominal mean pressure, $p_{m}$, and mean temperature, $T_{m}$, to yield the values of " $C_{p}$ (observed)" appearing in column 9.

The values of " $C_{p}$ (observed)" were plotted against the reciprocal of flow rate, and straight lines were fitted by least squares. Typical plots are given in figure 1, which shows the results at $10^{\circ} \mathrm{C}$. An analysis of variance, performed under the direction of W. J. Youden of the Bureau's Applied Mathematics Division, indicated that, for the data as a whole, the variation in slope of these lines was statistically significant. Consequently, the leastsquare intercepts have been retained in all cases as the desired value of heat capacity at each temperature and pressure, indicated as "extrapolated value" in table 1. The precision of the data is indicated by the value of $\sigma$, where $\sigma=\sqrt{\sum d^{2} /(n-1)}, d$ is the deviation of each point from the appropriate line, and $n$ is the number of experiments for each line. The average value of $\sigma$ is equivalent to \pm 0.03 percent of the heat capacity.

Results are summarized in table 2, having been converted to calories mole ${ }^{-1}$ degree $^{-1}$. (The defined calorie, equal to $4.1840 \mathrm{j}$, is used here.) A slight correction has been made, where necessary, for the 
TABLE 1. Data on perfluoropropane

\begin{tabular}{|c|c|c|c|c|c|c|c|c|}
\hline $\begin{array}{l}\text { Reciprocal } \\
\text { of rate, } F^{-1}\end{array}$ & $\begin{array}{l}\text { Mass of sample, } \\
m\end{array}$ & Power, $W$ & $\begin{array}{c}\text { Pressure drop, } \\
\qquad p\end{array}$ & $\begin{array}{l}\text { Temperature } \\
\text { rise, } \Delta T\end{array}$ & $\begin{array}{l}\text { Blank correc- } \\
\quad \text { tion, } \delta T\end{array}$ & Heat capacity, & $\begin{array}{l}\text { Correction for } \\
T_{m} \text { and } p_{m}, \\
\qquad C\end{array}$ & $\begin{array}{l}\text { Heat capacity, } \\
C_{p} \text { (observed) }\end{array}$ \\
\hline \multicolumn{9}{|c|}{$-30.00^{\circ} \mathrm{C}=243.16^{\circ} \mathrm{K} ; 1.00 \mathrm{~atm}$} \\
\hline $\begin{array}{c}s e c g^{-1} \\
9.1325 \\
10.4708 \\
11.7710 \\
14.8430 \\
17.8982 \\
28.3768 \\
28.8342\end{array}$ & $\begin{array}{c}g \\
57.5097 \\
71.9782 \\
65.1171 \\
88.1819 \\
46.0844 \\
44.6435 \\
38.2730\end{array}$ & $\begin{array}{c}w \\
0.753506 \\
.612918 \\
.615517 \\
.491159 \\
.413501 \\
.248342 \\
.247685\end{array}$ & $\begin{array}{c}m m \mathrm{Hg} \\
74.42 \\
55.83 \\
44.20 \\
28.41 \\
19.17 \\
7.14 \\
7.37\end{array}$ & $\begin{array}{r}\circ K \\
9.5427 \\
8.9149 \\
10.0930 \\
10.1797 \\
10.3433 \\
9.8719 \\
10.0048\end{array}$ & $\begin{array}{c}\circ K \\
0.1208 \\
.0933 \\
.0757 \\
.0517 \\
.0367 \\
.0150 \\
.0162\end{array}$ & $\begin{array}{c}j g^{-1} \mathrm{deg}^{-1} \\
0.71210 \\
.71243 \\
.71250 \\
.71254 \\
.71300 \\
.71278 \\
.71268\end{array}$ & $\begin{array}{r}j g^{-1} \mathrm{deg}^{-1} \\
+0.00038 \\
+.00031 \\
+.00003 \\
-.00017 \\
-.00037 \\
-.00024 \\
-.00018\end{array}$ & $\begin{array}{c}j g^{-1} \mathrm{deg}^{-1} \\
0.71248 \\
.71274 \\
.71253 \\
.71237 \\
.71263 \\
.71254 \\
.71250\end{array}$ \\
\hline \multicolumn{8}{|c|}{ Extrapolated value $\ldots . .}$. & $\begin{array}{r}0.71258 \\
\pm 0.00010\end{array}$ \\
\hline \multicolumn{9}{|c|}{$0.67 \mathrm{~atm}$} \\
\hline $\begin{array}{r}9.2277 \\
11.6910 \\
14.6602 \\
17.4329 \\
28.0026 \\
29.7293\end{array}$ & $\begin{array}{r}108.1488 \\
104.6250 \\
43.3911 \\
50.1947 \\
41.4276 \\
38.4368\end{array}$ & $\begin{array}{r}0.750862 \\
.614240 \\
.491139 \\
.411872 \\
.247657 \\
.248370\end{array}$ & $\begin{array}{r}111.95 \\
65.89 \\
42.97 \\
30.45 \\
11.73 \\
10.98\end{array}$ & $\begin{array}{r}9.6622 \\
10.0704 \\
10.1400 \\
10.1267 \\
9.8091 \\
10.4511\end{array}$ & $\begin{array}{r}0.1648 \\
.1036 \\
.0733 \\
.0567 \\
.0318 \\
.0305\end{array}$ & $\begin{array}{r}0.70506 \\
.70583 \\
.70498 \\
.70508 \\
.70471 \\
.70446\end{array}$ & $\begin{array}{r}+0.00043 \\
-.00027 \\
-.00003 \\
-.00001 \\
-.00012 \\
-.00033\end{array}$ & $\begin{array}{r}0.70549 \\
.70556 \\
.70495 \\
.70507 \\
.70459 \\
.70413\end{array}$ \\
\hline \multicolumn{8}{|c|}{ Extrapolated value } & $\begin{array}{r}0.70607 \\
\pm 0.00018\end{array}$ \\
\hline \multicolumn{9}{|c|}{$0.33 \mathrm{~atm}$} \\
\hline $\begin{array}{l}12.0251 \\
14.8191 \\
14.8249 \\
17.1889 \\
28.8417\end{array}$ & $\begin{array}{l}81.4694 \\
62.4984 \\
58.9523 \\
55.1834 \\
30.3918\end{array}$ & $\begin{array}{r}0.614151 \\
.491274 \\
.489272 \\
.412296 \\
.246727\end{array}$ & $\begin{array}{r}140.00 \\
84.97 \\
82.40 \\
61.64 \\
22.79\end{array}$ & $\begin{array}{l}10.3706 \\
10.2924 \\
10.2445 \\
10.0468 \\
10.1415\end{array}$ & $\begin{array}{r}0.1933 \\
.1287 \\
.1254 \\
.0987 \\
.0425\end{array}$ & $\begin{array}{r}0.69910 \\
.69861 \\
.69947 \\
.69857 \\
.69874\end{array}$ & $\begin{array}{r}-0.00018 \\
-.00044 \\
-.00054 \\
-.00027 \\
-.00017\end{array}$ & $\begin{array}{r}0.69892 \\
.69817 \\
.69893 \\
.69830 \\
.69857\end{array}$ \\
\hline \multicolumn{8}{|c|}{ Extrapolated value } & $\begin{array}{r}0.69875 \\
\pm 0.00034\end{array}$ \\
\hline \multicolumn{9}{|c|}{$+10.00^{\circ} \mathrm{C}=283.16^{\circ} \mathrm{K} ; 1.50 \mathrm{~atm}$} \\
\hline $\begin{array}{r}7.6341 \\
11.9426 \\
15.5622 \\
24.0445 \\
31.7895\end{array}$ & $\begin{array}{l}88.4219 \\
63.3854 \\
38.0904 \\
39.9908 \\
32.1109\end{array}$ & $\begin{array}{r}0.964866 \\
.616531 \\
.494454 \\
.328796 \\
.247218\end{array}$ & $\begin{array}{r}80.32 \\
32.92 \\
19.41 \\
8.25 \\
4.68\end{array}$ & $\begin{array}{r}9.4063 \\
9.4435 \\
9.8831 \\
10.1606 \\
10.0937\end{array}$ & $\begin{array}{r}0.0803 \\
.0345 \\
.0226 \\
.0150 \\
.0133\end{array}$ & $\begin{array}{r}0.77645 \\
.77685 \\
.77680 \\
.77693 \\
.77758\end{array}$ & $\begin{array}{r}+0.00060 \\
+.00058 \\
+.00029 \\
+.00002 \\
+.00006\end{array}$ & $\begin{array}{r}0.77705 \\
.77743 \\
.77709 \\
.77695 \\
.77764\end{array}$ \\
\hline \multicolumn{8}{|c|}{ Extrapolated value...... } & $\begin{array}{r}0.77699 \\
\pm 0.00026\end{array}$ \\
\hline \multicolumn{9}{|c|}{$1.00 \mathrm{~atm}$} \\
\hline $\begin{array}{r}7.8857 \\
12.1578 \\
15.4957 \\
18.4080 \\
23.2923 \\
31.4505\end{array}$ & $\begin{array}{l}78.2654 \\
44.1411 \\
51.0117 \\
47.5825 \\
43.5259 \\
35.7164\end{array}$ & $\begin{array}{r}0.963962 \\
.616923 \\
.493985 \\
.414090 \\
.328493 \\
.247232\end{array}$ & $\begin{array}{r}119.17 \\
49.72 \\
29.93 \\
21.82 \\
13.28 \\
7.26\end{array}$ & $\begin{array}{r}9.7483 \\
6.6800 \\
9.8900 \\
9.8584 \\
9.8988 \\
10.0655\end{array}$ & $\begin{array}{r}0.1103 \\
.0489 \\
.0270 \\
.0194 \\
.0131 \\
.0094\end{array}$ & $\begin{array}{r}0.77106 \\
.77094 \\
.77187 \\
.77169 \\
.77194 \\
.77178\end{array}$ & $\begin{array}{r}+0.00043 \\
+.00053 \\
+.00032 \\
+.00040 \\
+.00004 \\
+.00008\end{array}$ & $\begin{array}{r}0.77149 \\
.77147 \\
.77219 \\
.77209 \\
.77198 \\
.77170\end{array}$ \\
\hline \multicolumn{8}{|c|}{ Extrapolated value } & $\begin{array}{r}0.77162 \\
\pm 0.00030\end{array}$ \\
\hline \multicolumn{9}{|c|}{$0.50 \mathrm{~atm}$} \\
\hline $\begin{array}{r}9.5808 \\
15.7018 \\
23.6735 \\
31.9499\end{array}$ & $\begin{array}{l}73,4262 \\
41.9239 \\
38.3678 \\
33.6054\end{array}$ & $\begin{array}{r}0.617011 \\
.493877 \\
.329408 \\
.246343\end{array}$ & $\begin{array}{r}161.50 \\
59.04 \\
26.08 \\
14.49\end{array}$ & $\begin{array}{r}7.5868 \\
10.0547 \\
10.1422 \\
10.2447\end{array}$ & $\begin{array}{r}0.1406 \\
.0580 \\
.0269 \\
.0175\end{array}$ & $\begin{array}{r}0.76500 \\
.76683 \\
.76686 \\
.76695\end{array}$ & $\begin{array}{r}+0.00186 \\
-.00010 \\
-.00020 \\
-.00023\end{array}$ & $\begin{array}{r}0.76686 \\
.76673 \\
.76666 \\
.76672\end{array}$ \\
\hline & & & & & & \multicolumn{2}{|c|}{ Extrapolated value } & $\begin{array}{r}\text { 0. } 76687 \\
\pm 0.00006\end{array}$ \\
\hline
\end{tabular}


TABLE 1. Data on perfluoropropane-Continued

\begin{tabular}{|c|c|c|c|c|c|c|c|c|}
\hline $\begin{array}{l}\text { Reciprocal } \\
\text { of rate, } F^{-1}\end{array}$ & $\begin{array}{c}\text { Mass of sample, } \\
m\end{array}$ & Power, $W$ & $\begin{array}{l}\text { Pressure drop, } \\
\qquad p\end{array}$ & $\begin{array}{l}\text { Temperature } \\
\quad \text { rise, } \Delta T\end{array}$ & $\begin{array}{l}\text { Blank correc- } \\
\text { tion, }(T\end{array}$ & Heat capacity, & $\begin{array}{l}\text { Correction for } \\
T_{m} \text { and } p_{m}, \\
\qquad \Delta C\end{array}$ & $\begin{array}{l}\text { Heat capacity, } \\
C^{-} \text {(observed) }\end{array}$ \\
\hline \multicolumn{9}{|c|}{$+50.00^{\circ} \mathrm{C}=323.16^{\circ} \mathrm{K} ; 1.50 \mathrm{~atm}$} \\
\hline $\begin{array}{l}\sec g^{-1} \\
8.3615 \\
16.7869 \\
24.0516 \\
32.5992\end{array}$ & $\begin{array}{l}g \\
69.1872 \\
52.7321 \\
28.5482 \\
22.6392\end{array}$ & $\begin{array}{c}w \\
0.973454 \\
.4812918 \\
.345982 \\
.243888\end{array}$ & $\begin{array}{c}m m \mathrm{Hg} \\
77.59 \\
19.69 \\
9.69 \\
5.43\end{array}$ & $\begin{array}{l}\circ K \\
9.7307 \\
9.7010 \\
9.9928 \\
0.5597\end{array}$ & $\begin{array}{r}\circ K \\
0.0508 \\
.0146 \\
.0076 \\
.0045\end{array}$ & $\begin{array}{c}j g^{-1} \mathrm{deg}^{-1} \\
0.83214 \\
.83159 \\
.83211 \\
.83128\end{array}$ & $\begin{array}{r}j g^{-1} \mathrm{deg}^{-1} \\
+0.00034 \\
+.00034 \\
+.00014 \\
+.00044\end{array}$ & $\begin{array}{r}j g^{-1} \mathrm{deg}^{-1} \\
0.83248 \\
.83193 \\
.83225 \\
.83172\end{array}$ \\
\hline \multicolumn{8}{|c|}{$\underset{\sigma_{-}}{\text {Extrapolated value }}$} & $\begin{array}{r}0.83261 \\
\pm 0.00021\end{array}$ \\
\hline \multicolumn{9}{|c|}{$1.00 \mathrm{~atm}$} \\
\hline $\begin{array}{r}8.5834 \\
16.9441 \\
23.8000 \\
36.1370\end{array}$ & $\begin{array}{l}63.0260 \\
27.7200 \\
33.9298 \\
35.5804\end{array}$ & $\begin{array}{r}0.973891 \\
.481379 \\
.345495 \\
.244058\end{array}$ & $\begin{array}{r}112.88 \\
29.27 \\
14.99 \\
6.69\end{array}$ & $\begin{array}{r}01.0070 \\
9.8184 \\
9.9039 \\
10.6325\end{array}$ & $\begin{array}{r}0.0714 \\
.0209 \\
.0125 \\
.0070\end{array}$ & $\begin{array}{r}0.82943 \\
.82897 \\
.82921 \\
.82894\end{array}$ & $\begin{array}{r}+0.00008 \\
+.00017 \\
+.00011 \\
-.00039\end{array}$ & $\begin{array}{l}0.82951 \\
.82914 \\
.82932 \\
.82855\end{array}$ \\
\hline \multicolumn{8}{|c|}{$\underset{\sigma}{\text { Extrapolated value }}$} & $\begin{array}{r}0.82981 \\
\pm 0.00019\end{array}$ \\
\hline \multicolumn{9}{|c|}{$0.50 \mathrm{~atm}$} \\
\hline $\begin{array}{l}12.1633 \\
17.2428 \\
25.2559 \\
34.7789\end{array}$ & $\begin{array}{r}116.3520 \\
82.3232 \\
31.9494 \\
21.7759\end{array}$ & $\begin{array}{r}0.687173 \\
.481102 \\
.345631 \\
.243559\end{array}$ & $\begin{array}{r}117.66 \\
58.93 \\
27.28 \\
14.02\end{array}$ & $\begin{array}{l}\text { 10. } 0371 \\
\text { 10. } 0040 \\
10.5514 \\
10.2411\end{array}$ & $\begin{array}{r}0.0752 \\
.0407 \\
.0205 \\
.0114\end{array}$ & $\begin{array}{r}0.82654 \\
.82586 \\
.82570 \\
.82621\end{array}$ & $\begin{array}{r}+0.00011 \\
+.00020 \\
-.00020 \\
-.00011\end{array}$ & $\begin{array}{r}0.82665 \\
.82606 \\
.82550 \\
.82610\end{array}$ \\
\hline \multicolumn{8}{|c|}{$\begin{array}{r}\text { Extrapolated value_. } \\
\sigma_{--}\end{array}$} & $\begin{array}{r}0.82662 \\
\pm 0.00042\end{array}$ \\
\hline \multicolumn{9}{|c|}{$+90.00^{\circ} \mathrm{C}=363.16^{\circ} \mathrm{K} ; 1.50 \mathrm{~atm}$} \\
\hline $\begin{array}{r}8.2178 \\
16.3840 \\
23.8928 \\
35.7239\end{array}$ & $\begin{array}{l}89.1452 \\
56.9990 \\
29.8241 \\
24.9024\end{array}$ & $\begin{array}{r}1.088710 \\
0.542204 \\
.362959 \\
.272077\end{array}$ & $\begin{array}{r}91.27 \\
23.18 \\
11.04 \\
5.53\end{array}$ & $\begin{array}{r}10.0689 \\
10.0315 \\
9.8054 \\
10.9996\end{array}$ & $\begin{array}{r}0.0350 \\
.0083 \\
.0035 \\
.0013\end{array}$ & $\begin{array}{r}0.88548 \\
.88482 \\
.88410 \\
.88353\end{array}$ & $\begin{array}{r}+0.00015 \\
+.00009 \\
+.00024 \\
-.00048\end{array}$ & $\begin{array}{l}0.88563 \\
.88491 \\
.88434 \\
.88305\end{array}$ \\
\hline \multicolumn{8}{|c|}{$\begin{array}{r}\text { Extrapolated value_- } \\
\sigma_{--}\end{array}$} & $\begin{array}{r}0.88644 \\
\pm 0.00009\end{array}$ \\
\hline \multicolumn{9}{|c|}{$1.00 \mathrm{~atm}$} \\
\hline $\begin{array}{r}8.2735 \\
17.0922 \\
25.8957 \\
32.5131\end{array}$ & $\begin{array}{l}79.4052 \\
45.7350 \\
40.1121 \\
24.8082\end{array}$ & $\begin{array}{r}1.088739 \\
0.542399 \\
.363091 \\
.272998\end{array}$ & $\begin{array}{r}137.93 \\
32.51 \\
14.50 \\
9.27\end{array}$ & $\begin{array}{l}\text { 10. } 1385 \\
10.4810 \\
10.6396 \\
10.0527\end{array}$ & $\begin{array}{r}0.0563 \\
.0108 \\
.0028 \\
.0006\end{array}$ & $\begin{array}{r}0.88356 \\
.88362 \\
.88349 \\
.88290\end{array}$ & $\begin{array}{r}-0.00008 \\
-.00037 \\
-.00046 \\
-.00008\end{array}$ & $\begin{array}{r}0.88348 \\
.88325 \\
.88303 \\
.88282\end{array}$ \\
\hline \multicolumn{8}{|c|}{$\begin{array}{r}\text { Extrapolated value } \\
\sigma_{--}\end{array}$} & $\begin{array}{r}0.88371 \\
\pm 0.00002\end{array}$ \\
\hline \multicolumn{9}{|c|}{$0.50 \mathrm{~atm}$} \\
\hline $\begin{array}{l}11.8229 \\
16.7215 \\
24.4192 \\
33.7577\end{array}$ & $\begin{array}{l}\text { 91. } 7568 \\
61.3767 \\
34.9221 \\
28.0348\end{array}$ & $\begin{array}{r}0.768290 \\
.540098 \\
.362947 \\
.272367\end{array}$ & $\begin{array}{r}137.67 \\
69.10 \\
32.21 \\
17.42\end{array}$ & $\begin{array}{l}10.2451 \\
10.2177 \\
10.0491 \\
10.4279\end{array}$ & $\begin{array}{r}0.0591 \\
.0303 \\
.0094 \\
.0055\end{array}$ & $\begin{array}{r}0.88152 \\
.88127 \\
.88113 \\
.88125\end{array}$ & $\begin{array}{r}-0.00022 \\
-.00020 \\
-.00008 \\
-.00028\end{array}$ & $\begin{array}{l}0.88130 \\
.88107 \\
.88105 \\
.88097\end{array}$ \\
\hline \multicolumn{8}{|c|}{ Extrapolated value } & $\begin{array}{r}0.88138 \\
\pm 0.00007\end{array}$ \\
\hline
\end{tabular}




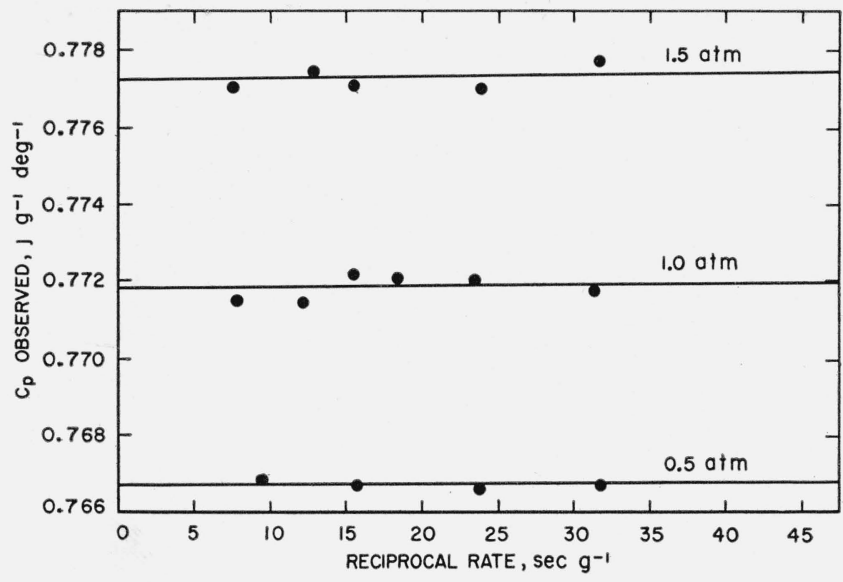

Figure 1. Measured heat-capacity values for perfluoropropane at $10^{\circ} \mathrm{C}$ and three pressures.

effect of curvature of the heat capacity-temperature function. Values of the heat capacity of the ideal gas $\mathrm{C}_{3} \mathrm{~F}_{8}$ were obtained by extrapolation of the results to zero pressure at each temperature of measurement, and are given as " $C_{p}^{0}$, observed" in table 2 .

There has been no complete assignment of the vibration fundamentals of perfluoropropane, and consequently statistical calculation of the ideal-gas heat capacity cannot yet be made. Also, there are no density data available for $\mathrm{C}_{3} \mathrm{~F}_{8}$ gas, so the values of the pressure coefficient of heat capacity, given in the last line of table 2, cannot yet be used in determining the constants of a low-pressure equation of state.

The experimental values of $C_{p}^{0}$ for hexafluoroethane (see footnote 1), perfluoropropane, and perfluorocyclobutane (see footnote 2), together with a calculated value ${ }^{5}$ for carbon tetrafluoride, are compared at one temperature $\left(50^{\circ}\right)$ in figure 2 by plotting against molecular weight. Similar plots are obtained at other temperatures. It is seen that a nearly constant increment of heat capacity per mole (amounting to about $10.3 \mathrm{cal} \mathrm{deg}^{-1} \mathrm{~mole}^{-1}$ at $25^{\circ} \mathrm{C}$ ) may be indicated for each added $\mathrm{CF}_{2}$ group, at least

s Rita M. Potocki and D. E. Mann (informal communication).

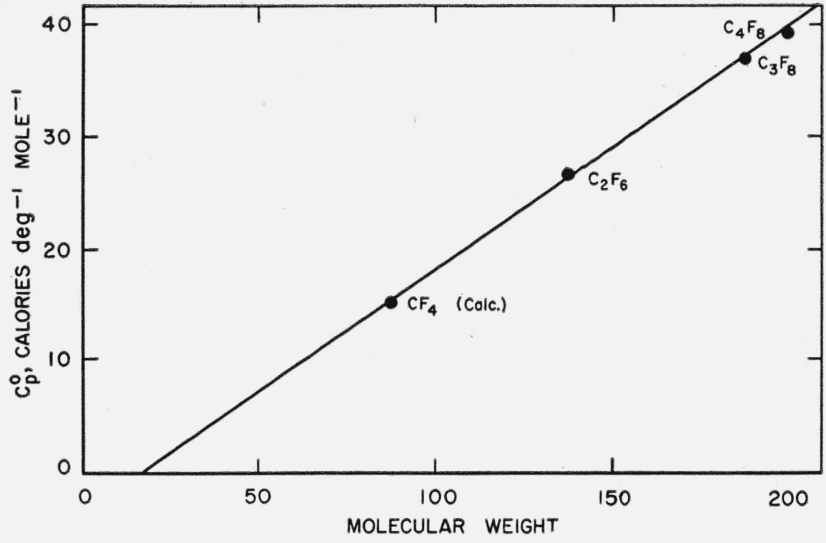

FIGURE 2. Variation with molecular weight of the ideal-yas heat capacities al $50^{\circ} \mathrm{C}$ of four saturaied fluorocarbons.

TABLE 2. Heat capacity of gaseous perfluoropropane $\left(\mathrm{C}_{3} \mathrm{~F}_{8}\right)$ Summary of results

\begin{tabular}{|c|c|c|c|c|}
\hline \multirow[t]{2}{*}{ Temperature, ${ }^{\circ} \mathrm{C}$} & -30.00 & +10.00 & +50.00 & +90.00 \\
\hline & \multicolumn{4}{|c|}{ Calories mole ${ }^{-1}$ degree $^{-1}$} \\
\hline \multirow{3}{*}{$\begin{array}{l}C_{p}, 1.50 \mathrm{~atm} \\
1.00 \mathrm{~atm} \\
0.67 \mathrm{~atm} \\
0.50 \mathrm{~atm} \\
0.33 \mathrm{~atm}\end{array}$} & 32.02 & $\begin{array}{l}\text { 34. } 93 \\
34.68\end{array}$ & $\begin{array}{l}37.42 \\
37.29\end{array}$ & $\begin{array}{l}39.84 \\
39.71\end{array}$ \\
\hline & 31.73 & & & \\
\hline & 31.40 & 34.45 & 37.15 & 39.61 \\
\hline \multirow[t]{2}{*}{$C_{p}^{0}$, observed } & 31.10 & 34. 22 & 37.02 & 39.49 \\
\hline & \multicolumn{4}{|c|}{ Calories mole degree $^{-1} \mathrm{~atm}^{-1}$} \\
\hline$\Delta C_{p} / \Delta P$ & 0.93 & 0.47 & 0.27 & 0.23 \\
\hline
\end{tabular}

in the straight-chain series. In the case of paraffin hydrocarbons, constancy of heat-capacity increments per added $\mathrm{CH}_{2}$ group occurs in the higher members of the series, beginning at about $n$-hexane.

Washington, September 29, 1953. 